\title{
Monocyte 'Reprogramming' and Mortality in Septic Patients with Acute Kidney Injury
}

\author{
Vladimír Tesař \\ Department of Nephrology, 1st School of Medicine, Charles University, Prague, Czech Republic
}

Sepsis is characterized by the systemic inflammatory response syndrome (SIRS), part of which is the activation of innate immunity and the 'cytokine storm'. This may ultimately result in multiple organ dysfunction syndrome, frequently involving acute kidney injury (AKI) with a high mortality rate usually surpassing $50 \%$.

In the last several years, significant progress has been made towards elucidation of the molecular mechanisms of SIRS [1]. Components of the bacterial wall, e.g. lipopolysaccharide (LPS) in gram-negative bacteria and lipoteichoic acid in gram-positive ones, stimulate the pathways of the innate immune system through the activation of the Toll-like receptors 4 and 2, respectively. The subsequent signaling is mediated by different protein kinases (including IRAK, JNK, p38 and ERK), resulting finally in the activation of the proinflammatory transcription factors nuclear factor- $\mathrm{\kappa B}(\mathrm{NF}-\kappa \mathrm{B})$ and activation protein-1. These transcription factors then stimulate the production of proinflammatory cytokines (e.g. IL-1, TNF, IL-12, migration inhibitory factor), chemokines (e.g. IL-8/ CXCL8, or CCL5), their receptors, adhesion molecules and eicosanoids with potential damage to the body tissues and organs, including the lung and the kidney.

More recently, it has been clearly demonstrated that this dysregulated immune activation is frequently accompanied by the clinical and laboratory signs of depressed immune status [2], e.g. increased susceptibility to certain infections, anergy to skin antigens and reduced

\section{KARGER}

Fax +4161306 1234 E-Mail karger@karger.ch www.karger.com (c) 2008 S. Karger AG, Basel

0253-5068/08/0262-0186\$24.50/0

Accessible online at:

www.karger.com/bpu expression of leukocyte surface markers (HLA-DR antigens, chemokine receptors, e.g. CXCR2) with reduced antigen presentation, depressed cytokine production and diminished cellular cytotoxicity (leukocyte 'reprogramming'). Although the mechanisms of this 'immune paralysis' have yet to be fully elucidated, both impaired NF$\kappa \mathrm{B}$ activation and activation of heat-shock proteins were suggested to play an important role.

Sepsis is also accompanied by oxidative stress (increased production of reactive oxygen species) with the dissipation of the mitochondrial inner membrane potential and release of cytochrome c into the cytosol, resulting in the activation of the intrinsic caspase system and programmed cell death. Oxidative stress may also augment the activation of NF- $\mathrm{\kappa B}$ and alter the structure of the lipids and proteins of the lipid rafts (including the expression of Toll-like receptor 4 [3]), which is essential for LPS signaling, thus contributing to monocyte reprogramming [4].

Persistently decreased monocyte HLA-DR expression was shown to predict mortality in infections complicating trauma [5], in severe community-acquired infections [6], or in patients with septic shock [7], but these data were not confirmed in another large group of septic patients [8].

The paper of Silva et al. in this issue is the first report on the relation between HLA-DR expression and the outcome of septic patients with severe AKI requiring 
CRRT. Although septic patients with AKI had higher AOPP and a lower percentage of monocytes expressing HLA-DR (with no correlation between these two markers) compared to healthy controls, there was - despite the trend - no significant difference in AOPP levels and HLA-DR expression between survivors and non-survivors. Despite some limitations (relatively small study, no other avaiable markers of oxidative stress and immune markers except AOPP and HLA-DR) which preclude a definitive conclusion, a relation between these markers and the outcome of the septic patients with AKI, if any, may be only weak, and so, in any case, probably not very useful for clinical practice.

Having in mind the poor outcome of septic patients with AKI, further studies dedicated both to the better understanding of immune dysregulation in sepsis and sepsis-related AKI and the identification of more useful prognostic markers and targets for new therapeutic approaches are clearly warranted.

\section{References}

1 Matsuda N, Hattori Y: Systemic inflammatory response syndrome (SIRS): molecular pathophysiology and gene therapy. J Pharmacol Sci 2006;101:189-198.

-2 Dobrovolskaia MA, Vogel SN: Toll receptors, CD14, and macrophage activation and deactivation by LPS. Microbes Infect 2002;4: 903-914.

3 Powers KA, Szászi K, Khadaroo RG, et al: Oxidative stress generated by hemorrhagic shock recruits Toll-like receptor 4 to the plasma membrane in macrophages. J Exp Med 2006;203:1951-1961.
-4 Cuschieri J, Maier RV: Oxidative stress, lipid rafts, and macrophage reprogramming. Antioxid Redox Signal 2007;9:1485-1497.

5 Hershman MJ, Cheadle WG, Wellhausen SR, et al: Monocyte HLA-DR antigen expression characterizes clinical outcome in the trauma patients. Br J Surg 1990;77:204-207.

6 6 Lekkou A, Karakantza M, Mouzaki A, et al: Cytokine production and monocyte HLADR expression as predictors of outcome for patients with community-acquired severe infections. Clin Diagn Lab Immunol 2004; 11:161-167.
7 Monneret G, Finck ME, Venet F, et al: The anti-inflammatory response dominates after septic shock: association of low monocyte HLA-DR expression and high interleukin-10 concentration. Immunol Lett 2004;95:193198.

8 Perry SE, Mostafa SM, Wenstone R, et al: Is low monocyte HLA-DR expression helpful to predict outcome in severe sepsis? Intensive Care Med 2003;29:1245-1252. 